\title{
Evaluation prevalence of Pompe disease in Iranian patients with myopathies of unknown etiology
}

\author{
Khadijeh Haji Naghi Tehrani ${ }^{1}$, Elmira Sakhaeyan², Elnaz Sakhaeyan ${ }^{3}$
}

${ }^{1}$ M.D., Neurologist, Assistant Professor, Department of Neurology, Islamic Azad University, Tehran Medical Branch, Tehran, Iran

${ }^{2}$ M.D., Graduated from Islamic Azad University, Tehran Medical Branch, Tehran, Iran

${ }^{3}$ Pharm.D., Graduated from Tehran University of Medical Science (TUMS), Tehran, Iran

\section{Type of article: Original}

\begin{abstract}
Background: Pompe disease is a rare but potentially treatable metabolic disorder having an estimated worldwide incidence of one in forty thousand live births. While the introduction of enzyme replacement therapy (ERT) has considerably increased the awareness of the disease, the delay in diagnosis is still consistent and most patients go undetected.

Objective: This study aimed to determine the prevalence of late-onset Pompe disease (LOPD) in a high-risk population, using dried blood spot (DBS) as a main screening tool.

Methods: This cross-sectional study was performed on the 93 patients who attended to the neuromuscular center of Bu-ali hospital in Tehran, Iran, during 2014-2015. Inclusion criteria were: 1) age $\geq 1$ years, 2) proximal myopathies of unknown etiology in lower limbs or symptoms of limb girdle muscle weakness (LGMW), and 3) unexplained elevated CPK (>174). Acid $\alpha$-glucosidase (GAA) activity was measured separately on DBS by fluorometric method. For the final diagnosis, GAA deficiency was confirmed by a biochemical assay in skeletal muscle, whereas genotype was assessed by GAA molecular analysis. All statistical tests were performed using the SPSS version 16. Results are presented as mean (SD) or median (IQR), as appropriate.

Results: In a 12-month period, we studied 93 cases: 5 positive samples $(5.3 \%)$ were detected by DBS screening, biochemical and molecular genetic studies finally confirmed LOPD diagnosis in 3 cases (3.22\%). Among the 93 patients, 100\% showed hyperCKemia, 89 patients (95.7\%) showed LGMW and 4 patients had symptoms of proximal myopathies in the lower limb.

Conclusions: Results from the LOPED study suggest that GAA activity requires accurate screening by DBS in all patients referred for hyperCKemia and/or LGMW.

Keywords: Pompe disease, Proximal myopathy, hyperCKemia
\end{abstract}

\section{Introduction}

Pompe disease, also called glycogen storage disease type II, (GSD II) is a rare autosomal recessive disorder due to acid $\alpha$-glucosidase (GAA) deficiency leading to glycogen accumulation in several tissues, with predilection of skeletal muscle and heart (1). Clinically, it is associated with a range of phenotypes, with variable organ involvement, age of onset and severity degree (2). Late-onset Pompe disease (LOPD) is a slowly progressive form with juvenile or adult presentation, characterized by progressive muscle weakness and often, respiratory impairment (3). Diagnosis of LOPD is still challenging and often quite delayed. This has been hypothesized to be due to several reasons such as rarity of the disorder, wide clinical spectrum, overlap of signs and symptoms with other neuromuscular disorders, or variable diagnostic approach in different countries (4). Recently, Kishnani et al., (5) using data from the International Pompe Registry, calculated the time interval between onset and diagnosis, 'a diagnostic gap', in different categories of patients with Pompe disease, and still found this delay consistent. First carried out in 2006, enzyme replacement therapy (ERT) represents the first disease-specific treatment. In patients

\section{Corresponding author:}

Assistant Professor Dr. Khadijeh Haji Naghi Tehrani, Department of Neurology, Islamic Azad University, Tehran Medical Branch, Tehran, Iran. Tel: +98(912)1493324, Email: dr_tehrani10@yahoo.com

Received: May 24, 2016, Accepted: September 26, 2016, Published: July 2017

iThenticate screening: September 29, 2016, English editing: May 12, 2017, Quality control: June 16, 2017

(C) 2017 The Authors. This is an open access article under the terms of the Creative Commons Attribution-NonCommercialNoDerivs License, which permits use and distribution in any medium, provided the original work is properly cited, the use is non-commercial and no modifications or adaptations are made. 
with LOPD, ERT was less effective in older juveniles and adults than in infants, likely because of delayed diagnosis $(4,6)$. These considerations suggested development of more rapid diagnostic tools, such as the dried blood spot (DBS), to detect GAA activity, which could result in an earlier LOPD diagnosis (7-10). To the best of our knowledge, there are study that estimated the prevalence of LOPD among the Iranian population. This study was aimed to determine the prevalence of late-onset Pompe disease (LOPD) in a high-risk population, using the dried blood spot (DBS) as a main screening tool.

\section{Material and Methods}

\subsection{Research design and setting}

This cross-sectional study was performed on patients with suspected neuromuscular disorders who attended the neuromuscular center of Bu-ali hospital from 1 January 2014 to December 2015, in Tehran, Iran. The study was approved by the ethical committee of Islamic Azad University, Tehran Medical Sciences Branch, and written informed consent for participation was obtained.

\subsection{Sampling}

Regarding the study type, all the patients who attended the neuromuscular center of Bu-ali hospital were consecutively enrolled into the study, based on the inclusion criteria.

\subsection{Inclusion and exclusion criteria}

Inclusion criteria were 1) age >= year old, 2) proximal myopathies of unknown etiology in lower limbs or symptoms of limb girdle muscle weakness (LGMW), and 3) unexplained elevated CPK (CPK>174). Exclusion criteria were: 1) age $<1$ year old, and 2) myopathies with known or explained etiology. Blood samples were collected, immediately spotted on filter paper (DBS), and dried at room temperature.

\subsection{Data collection}

DBS samples were sent to laboratories specialized in metabolic disorders, to be examined by experts in measuring GAA activity, by fluorometry techniques. For each patient, clinical history and laboratory data were collected in a Case Report Form (CRF). GAA activity in DBS was assessed by a fluorometry method using the substrate 4methylumbelliferyl- $\alpha$-D-glucoside (11). The interval between the 99th centile activity of the disease range (5.78 $\mathrm{nM} / \mathrm{h} / \mathrm{mL})$ and the first centile activity of the normal controls $(6.10 \mathrm{nM} / \mathrm{h} / \mathrm{mL})$ was considered as the cut-of target range, and the cut-off for $\alpha$-glucosidase activity at $6 \mathrm{nM} / \mathrm{h} / \mathrm{mL}$ on DBS. Patients who were considered positive were those with GAA activity below $6 \mathrm{nM} / \mathrm{h} / \mathrm{mL}$.

\subsection{Statistical analysis}

All statistical tests were performed using the SPSS software package Version 16 (SPSS Inc, Chicago, Illinois, USA). Results are presented as mean (SD) or median (IQR), as appropriate. The patients, recruited from one Iranian neuromuscular center, were all admitted for diagnostic purposes.

\section{Results}

Of a total of 93 patients, 42 patients (45.2\%) were male, and 51 patients (54.8\%) were female. The mean age of the patients was $27 \pm 11.02$ years. All the patients presented with hyperCKemia, $95.7 \%$ (89 patients) with LGMW and $4.3 \%$ (4 Patients) with proximal myopathy of the lower limbs. Of the recruited patients, $33(35.5 \%)$ had a positive family history of myopathy. Forty $(43 \%)$ of the recruited patients were children of consanguinity. In our study, five patients (three female and two male) were diagnosed with low level of acid alpha glucosidase activity. Only three out of five patients (one female and two male) received a positive genetic result as confirmation of Pompe disease. Hence, the Pompe disease prevalence was reported as $3.22 \%$. Using the fluorometric method, reduced GAA enzyme activity in DBS was found in five participants (5.3\%). A confirmatory biochemical test performed on skeletal muscles of the five patients resulted positive at the DBS testing; GAA deficiency was confirmed in $3 / 5$ patients $(3.22 \%)$. Among the three patients with LOPD, $1 / 3$ was female $(33.33 \%)$; the mean age at disease onset was 17.66 years, while the median age at disease diagnosis was 30.66 years. All three of these patients had hyperCKemia and proximal myopathy of the lower limbs.

\section{Discussion}

Pompe disease is a rare disorder having an estimated worldwide incidence of 1 in 40000 live births. However, several studies have suggested that incidence rates may vary among different ethnic populations with a fluctuating range from 1 in 14000 to 1 in 300000 (12). Since the introduction of ERT, either among neuromuscular experts or 
general practitioners, the awareness of this disease has considerably increased; but, unfortunately, the delay of diagnosis is still consistent, especially in LOPD (5), because of the rarity of the disorder and the variable clinical presentation or overlapping of symptoms with other neuromuscular disorders. The recent studies have tried to improve the approach to a rapid and correct diagnosis using the DBS method in targeted populations. The DBS GAA enzyme assay test is a robust, rapid and reliable first tier test for screening Pompe disease, thus it is recommended to be performed for patients with unexplained myopathies suspected of Pompe disease

1) Genetic confirmatory tests are recommended to confirm diagnosis of Pompe disease

2) Increased awareness of Pompe disease, clinical signs and symptoms and diagnostic algorythm is needed to help more timely diagnosis and to prevent irreversible sequels

3) With due attention to prevalence rate in our study, assessment of patients with symptoms of limb girdle myopathy /proximal myopathy plus unexplained elevated CPK -especially when orthopnea is present, is recommended

As shown in several studies, early diagnosis is of high importance in LOPD patients. The earlier the treatment is started, the better the outcome will be. In Taiwan, in 2005, a nationwide NBS program for Pompe disease was initiated with highly successful results. So far, a large number of newborns have been screened for Pompe disease (over 400000 infants). Of those screened, six infants were found to carry the infantile-onset Pompe disease (LOPDprevalence $1 / 57000)$, and 20 cases of LOPD and 294 cases of pseudo deficiency were also detected $(13,14)$. On the other hand, considered as key features, specific signs or symptoms, usually represented by hyperCKemia and/or unclassified LGMW, Pompe disease has been screened by DBS, although in a limited number of patients and in different ethnic populations. Recently, Spada et al. (11) screened 137 patients with unclassified hyperCKemia and found a 2.2\% prevalence of Pompe disease. A 2013 study by Preisler et al. (15) using the DBS method to evaluate the prevalence of Pompe disease in undetermined patients with limb-girdle muscular dystrophy, identified three patients with Pompe disease in 38 individuals screened (8\%). Consequently, in the present study, in a period of 12 months, we were able to collect 93 samples from patients with suspected neuromuscular disorders because of proximal LGMW with hyperCKemia. In this group of patients, we used the DBS assay as the main screening tool to check GAA activity: we found, five samples with low GAA activity on DBS, but after the biochemical confirmatory assay on skeletal muscle, LOPD diagnosis was assessed in three patients (3.22\%). Among those patients, $100 \%$ showed hyperCKemia, 95.7\% showed LGMW, and 4.3\% manifested with proximal myopathy of the lower limbs. The prevalence of Pompe disease is reported as 3.22\%. A 2006 study by Fernandez et al., (16) retrospectively reviewed muscle biopsy specimens of 104 patients with hyperCKemia and found Pompe disease in four patients (3.8\%). Although Vissing et al., (17) in a recent diagnostic review, indicated the 'blood-based' assays as a prevalent diagnostic tool, muscle biopsy still had an important role. The interpretation of DBS results needs great accuracy and experience. In fact, the results may indicate some false positives (9). This also happened in our study, considering that we first obtained five positive samples; finally, three patients were biochemically confirmed with a diagnosis of LOPD. A possible explanation of those slightly controversial results could be due to the quality of samples. It is well known that DBS specimen collection and storage can be crucial for a correct analysis (18).

\section{Conclusions}

The result of this study revealed that LOPD study suggests that GAA activity should be accurately screened by DBS in all patients referring for hyperCKemia and/or LGMW. However, future multicenter study with larger sample size should be performed to estimate the prevalence the LOPD among the Iranian population.

\section{Acknowledgments:}

This study was supported by Islamic Azad University, Tehran Medical Sciences Branch, Tehran, Iran. The funding organizations had no role in the design or conduct of the study; or the collection, management, or analysis of the data; or the preparation, review, or approval of the manuscript.

\section{Conflict of Interest:}

There is no conflict of interest to be declared.

\section{Authors' contributions:}

All authors contributed to this project and article equally. All authors read and approved the final manuscript.

\section{References:}

1) Hers HG. Alpha glucosidase deficiency in generalized storage disease (pompe's disease). Bochem J. 1963; 86: 11-16. doi: 10.1042/bj0860011. PMID: 13954110, PMCID: PMC1201703. 
2) Kishnani P, Hwu W, Mandel H, Nicolino M, Yong F, Corzo D. A retrospective, multinational, multicenter study on the natural history of infantile-onset Pompe disease. J Pediatr. 2006; 148: 671-6. doi: 10.1016/j.jpeds.2005.11.033. PMID: 16737883.

3) Gaeta M, Barca E, Ruggeri P, Minultoli F, Rodolico C, Mazziotti S, et al. Late onset pompe diseas (LOPD): correlations between respiratory muscles CT and MRI features and pulmonary function. Mol Genet Metab. 2013; 110: 290-6. doi: 10.1016/j.ymgme.2013.06.023. PMID: 23916420.

4) Toscano A, Montagnese F, Musumeci O. Early is better? A new algorithm for early diagnosis in late onset Pompe disease (LOPD). Acta Myol. 2013; 32(2): 78-81. PMCID: PMC3866896.

5) Kishnani PS, Amartino HM, Lindberg C, Miller TM, Wilson A, Keutzer J. Timing of diagnosis of patients with Pompe disease: data from the Pompe registry. Am J Med Genet A. 2013; 161: 2431-43. doi: 10.1002/ajmg.a.36110. PMID: 23997011.

6) Chien YH, Hwu WL, Lee NC. Pompe disease: early diagnosis and early treatment make a difference. Pediatr Neonatol. 2013; 54: 219-27. doi: 10.1016/j.pedneo.2013.03.009. PMID: 23632029.

7) Kallwass H, Carr C, Gerrein J, Titloe M, Pomponio R, Bali D, et al. Rapid diagnosis of late onset Pompe disease by fluorometric assay of alpha-glucosidase activities in dried blood spots. Mol Genet Metab. 2007; 90: 449-52. doi: 10.1016/j.ymgme.2006.12.006. PMID: 17270480.

8) La marca G, Casetta B, Malvagia S, Guerrini R, Zammarchi E. New strategy for screening of lysosomal storage dosorders:the use of online trapping-and-cleanup liquid chromatography/mass spectrometry. Anal Chem. 2009; 81(15): 6113-21. doi: 10.1021/ac900504s. PMID: 19555116.

9) Goldstein JL, Young SP, Changela M, Dickerson GH, Zhang H, Dai J, et al. Screening for Pompe disease using a rapid dried blood spot method: experience of a clinical diagnostic laboratory. Muscle Nerve. 2009; 40(1): 32-6. doi: 10.1002/mus.21376. PMID: 19533645.

10) Lukacs Z, Nieves Cobos P, Mengel E, Hartung R, Beck M, Deschauer M, et al. Diagnostic efficacy of the fluorometric determination of enzyme activity for Pompe disease from dried blood specimens compared with lymphocytes-possibilitynfor newborn screening. J Inherit Metab Dis. 2010; 33(1): 43-50. doi: 10.1007/s10545-009-9003-z. PMID: 20033296.

11) Spada M, Porta F, Vercelli L, Pagliardini V, Chiado-Piat L, Boffi P. Screening for later-onset Pompe's disease in patients with paucisymptomatic hyperCKaemia. Mol Genet Metab. 2013; 109: 171-3. doi: 10.1016/j.ymgme.2013.03.002. PMID: 23566438.

12) Ausems MG, Verbiest J, Hermans MP, Kroos MA, Beemer FA, Wokke JH, et al. Frequency of glycogen storage disease type II in The Netherlands: implications for diagnosis and genetic counselling. Eur J Hum Genet. 1999; 7(6): 713-6. doi: 10.1038/sj.ejhg.5200367. PMID: 10482961.

13) Chien YH, Chiang SC, Zhang XK, Keutzer J, Lee NC, Huang AC, et al. Early detection of Pompe disease by newborn screening is feasible: resulys from the Taiwan screening program. Pediatrics. 2008; 122(1): 3945. doi: 10.1542/peds.2007-2222. PMID: 18519449.

14) Yang CF, Liu HC, Hsu TR, Tsai FC, Chiang SF, Chiang CC, et al. A large-scale nationwide newborn screening program for Pompe disease in Taiwan: towards effective diagnosis and treatment. Am J Med Genet A. 2014; 164: 54-61. doi: 10.1002/ajmg.a.36197. PMID: 24243590.

15) Preisler N, Lukacs Z, Vinge L, Madseh KL, Hansen RS. Late-onset Pompe disease is prevalent in unclassified limb-girdle muscular dystrophies. Mol Gen Metab. 2013; 110(3): 287-9. doi: 10.1016/j.ymgme.2013.08.005. PMID: 24011652.

16) Frenandez C, de Paula A, Figarella-Branger D, Kranha M, Giorqi R. Diagnostic evaluation of clinically normal subjects with chronic hyperCKaemia. Neurology. 2006; 66: 1585-7. doi: 10.1212/01.wnl.0000216144.69630.6e. PMID: 16717227.

17) Vissing J, Lukacs Z, Straub V. Diagnosis of Pompe disease. Muscle biopsy vs blood-based assays. JAMA Neurol. 2013; 70: 923-7. doi: 10.1001/2013.jamaneurol.486. PMID: 23649721.

18) Pompe Disease Diagnostic Working Group, Winchester B, Bali D, Bodamer OA, Caillaud C, Christensen $\mathrm{E}$, et al. Methods for a prompt and reliable laboratory diagnosis of Pompe disease: report from an international consensus meeting. Mol Genet Metab. 2008; 93(3): 275-81. doi: 10.1016/j.ymgme.2007.09.006. PMID: 18078773. 